\title{
Non Data-Aided SNR Estimation of OFDM Signals
}

\author{
François-Xavier Socheleau, Abdeldjalil Aïssa-El-Bey, and Sébastien Houcke
}

\begin{abstract}
This letter deals with the problem of non data aided (NDA) signal to noise ratio (SNR) estimation of OFDM signals transmitted through unknown multipath fading channel. Most of existing OFDM SNR estimators are based on the knowledge of pilot sequences which is not applicable in some contexts such as cognitive radio for instance. We show that it is possible to take advantage of the periodic redundancy induced by the cyclic prefix to get an accurate NDA SNR estimator. Numerical simulations highlight the benefit of the proposed method compared with the state of the art.
\end{abstract}

Index Terms-SNR estimation, OFDM, non data-aided.

\section{INTRODUCTION}

$\mathbf{S}$ IGNAL to noise ratio (SNR) is an indicator commonly used to evaluate the quality of a communication link. More specifically, SNR knowledge enables wireless systems to improve propagation channel estimation and is a key decision parameter in adaptive processes such as dynamic reconfiguration of cognitive radios, adaptive modulation and coding (AMC) or adaptive power allocation.

Several OFDM SNR estimators have already been suggested [1], [2] but most of them are based on the pilot symbol knowledge. This kind of estimation method is commonly classified as Data-Aided (DA). DA algorithms proved their efficiency but cannot be applied in every context. For instance, in some cognitive radio applications, terminals need to sense the link quality with all the surrounding networks to decide which one is the most appropriate to communicate with. In this case, a cognitive terminal may not tolerate any delay in getting frame synchronized (operation required to know the pilots location) with each network before deciding which one is the most suited to its needs. To overcome DA method limitations, a Non Data Aided (NDA) SNR estimator for OFDM systems is introduced in [3]. To the best of our knowledge, this estimator is the only NDA algorithm for OFDM published to date. It can achieve good accuracy but its performance is based on a subjective choice of a threshold level. Moreover, the signal and noise power are not estimated independently which results in performance degradation at high SNR.

In this letter, we address these issues and exploit the correlation as well as the cyclostationarity induced by the cyclic-prefix (CP) to propose a new NDA SNR estimator of OFDM signals transmitted through unknown multipath fading channel.

Manuscript received July 18, 2008. The associate editor coordinating the review of this letter and approving it for publication was F. Jondral. This material is based upon work supported by Agence Nationale de la Recherche under grant "DEMAIN."

The authors are with Institut TELECOM; TELECOM Bretagne; UMR CNRS 3192 Lab-STICC, Université européenne de Bretagne, Technopôle Brest Iroise-CS 83818, 29238 Brest Cedex, France (e-mail: \{fx.socheleau, abdeldjalil.aissaelbey, sebastien.houcke\}@telecom-bretagne.eu).

Digital Object Identifier 10.1109/LCOMM.2008.081134

\section{OFDM Signal MODEL}

Assuming that an OFDM symbol consists of $N$ subcarriers, the discrete-time baseband equivalent transmitted signal is given by

$$
\begin{gathered}
x(m)=\sqrt{\frac{E_{s}}{N}} \sum_{k \in \mathbb{Z}} \sum_{n=0}^{N-1} a_{k}(n) e^{2 i \pi \frac{n}{N}(m-D-k(N+D))} \\
. g(m-k(N+D)),
\end{gathered}
$$

where $E_{s}$ is the signal power and $a_{k}(n)$ are the transmitted data symbols at the $n$-th subcarrier of the $k$-th OFDM block. These data symbols are assumed to be independent, identically distributed (i.i.d) and of unitary variance. $D$ is the CP length and $m \mapsto g(m)$ is the rectangular pulse shaping filter.

Let $\{h(l)\}_{l=0, \cdots, L}$ be a baseband equivalent discrete-time Rayleigh fading channel impulse response of length $L+1$ with $L+1<D$ such that $h(l) \sim \mathcal{C N}\left(0, \sigma_{h(l)}^{2}\right)$. The received samples of the OFDM signal are then expressed as

$$
y(m)=e^{-i\left(2 \pi \varepsilon \frac{m-\tau}{N}+\theta\right)} \sum_{l=0}^{L} h(l) x(m-l-\tau)+\eta(m),
$$

where $\varepsilon$ is the carrier frequency offset, $\theta$ the initial arbitrary carrier phase, $\tau$ the timing offset and $\eta(m)$ the additive white Gaussian noise such that $\eta(m) \sim \mathcal{C N}\left(0, \sigma_{\eta}^{2}\right)$.

The SNR is defined as

$$
\mathrm{SNR}=\frac{S}{\sigma_{\eta}^{2}}
$$

with

$$
S=E_{s} \sum_{l=0}^{L} \sigma_{h(l)}^{2} .
$$

\section{NOISE VARIANCE ESTIMATION}

Similarly to what suggested in [3], the noise variance can be estimated thanks to the redundancy induced by the CP. In fact, the $\mathrm{CP}$ use leads to $x(k(N+D)+m)=$ $x(k(N+D)+N+m), \forall k \in \mathbb{Z}$ and $\forall m \in\{0, \cdots, D-1\}$. It is then straightforward to see that if we assume perfect synchronization $^{1}$ at reception (i.e $\tau=0$ and $\epsilon=0$ ) and a time-invariant channel over an OFDM symbol duration, we can get $D-L$ noise variance estimates defined as

$$
\hat{\sigma}_{\eta, u}^{2}=J(u), L \leq u \leq D-1
$$

with

$$
\begin{aligned}
J(u)=\frac{1}{2 M(D-u)} \sum_{k=0}^{M-1} \sum_{m=u}^{D-1} \mid y(k(N+D)+m)- \\
\left.y(k(N+D)+N+m)\right|^{2}
\end{aligned}
$$

\footnotetext{
${ }^{1}$ for NDA OFDM synchronization algorithms refer to [4], [5]
} 
where $M$ denotes the number of OFDM symbols in the observation window. It can be easily shown that the estimator with the smallest variance is found for $u=L$. The difficulty is then to estimate $L$. Cui et al. suggested an estimator in [3] but it has the major disadvantage of being based on a threshold level chosen arbitrarily. To overcome this limitation we hereafter propose a method inspired by maximum likelihood estimation.

From Eq. (3), $J(u)$ can be expressed as

$$
J(u)=\left(1-\frac{1}{D-u}\right) J(u+1)+\xi(u)
$$

where $\xi(u)$ is a random variable which follows a chi square distribution for $L \leq u \leq D-1$. This distribution can be simplified, for $M$ large enough, as

$$
\xi(u) \sim \mathcal{N}\left(\frac{\sigma_{\eta}^{2}}{D-u}, \frac{\sigma_{\eta}^{4}}{M(D-u)^{2}}\right) .
$$

$L$ is then estimated using the likelihood function $f\left(\mathbf{X}_{u} \mid L=u\right)$ with $\mathbf{X}_{u}$ the observation variables defined as $\mathbf{X}_{u}=(\xi(u), \xi(u+1), \cdots, \xi(D-1))$. The different $\xi(u)$ being independent, $\hat{L}$ is given by

$\hat{L}=\underset{u}{\operatorname{argmax}}\left[\prod_{m=u}^{D-1} f(\xi(m) \mid L=u)\right]^{1 /(D-u)} \quad, 0 \leq u \leq D-1$

where $f(\xi(m) \mid L=u)$ is computed thanks to Eq. (4) by making the approximation that $\sigma_{\eta}^{2} \simeq J(u)$. Note that because the observations $\mathbf{X}_{u}$ are of variable lengths, Eq. (5) is defined as an average likelihood which is the geometric mean of the individual likelihood elements.

\section{Signal Power Estimation}

We here propose to use the cyclostationary statistics induced by the CP [6] to estimate the signal power. This kind of statistics is appealing as the noise is hardly never cyclostationary. More precisely, we base our estimator on the cyclic autocorrelation defined as [7]

$$
R_{x}^{\alpha}(u)=\lim _{M \rightarrow+\infty} \frac{1}{M} \sum_{m=0}^{M-1} \mathbb{E}\left[x(m) x^{*}(m+u)\right] e^{-2 i \pi m \alpha}
$$

where $\alpha$ is called the cycle frequency. From Eq. (1), it can be shown that

$$
\begin{aligned}
R_{x}^{\alpha}(N)= & E_{s} \frac{\sin (\pi \alpha D)}{(N+D) \sin (\pi \alpha)} e^{-i \pi \alpha(D-1)} . \\
& \sum_{q \in \mathbb{Z}} \delta\left(\alpha-\frac{q}{N+D}\right)
\end{aligned}
$$

where $\delta($.$) denotes the Kronecker symbol. Moreover, as$ proved in [6], the cyclic autocorrelation at reception writes

$$
R_{y}^{\alpha}(N)=R_{x}^{\alpha}(N) \sum_{l=0}^{L} \sigma_{h(l)}^{2} e^{-2 i \pi \frac{\alpha l}{N+D}} .
$$

Therefore, a signal power estimate can be given by

$$
\hat{S}=\frac{1}{2 N_{c}+1}\left|\sum_{q=-N_{c}}^{N_{c}} \hat{R}_{y}^{q \alpha_{0}}(N) \frac{\sin \left(\pi q \alpha_{0}\right)}{\alpha_{0} \sin \left(\pi q \alpha_{0} D\right)} e^{i \pi q \alpha_{0}(D-1)}\right|
$$

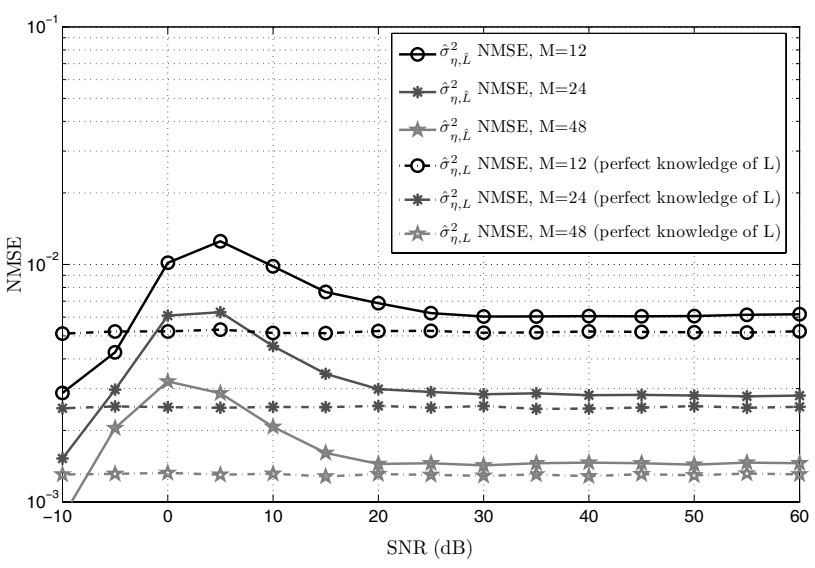

Fig. 1. NMSE of the noise variance estimator.

where $\alpha_{0}=1 /(N+D)$ and

$$
\hat{R}_{y}^{q \alpha_{0}}(N)=\frac{\sum_{m=0}^{M(N+D)-1} y(m) y^{*}(m+N) e^{-2 i \pi m q \alpha_{0}}}{M(N+D)} .
$$

$N_{c}$ represents the number of considered cycle frequencies to estimate the signal power. The choice of $N_{c}$ is a trade-off between the estimator bias and variance. From Eq. (8), it can be shown that the estimator asymptotic variance (i.e. for $M \rightarrow+\infty)$ decreases as the number of cycle frequencies increases. However, Eq. (6) indicates that it may be judicious to choose cycle frequencies within the first lob of $\hat{R}_{y}^{q \alpha_{0}}(N)$ as for $q \geq N / D$, the power of this function is very small. In addition, to limit the estimator bias, $N_{c}$ has to be bounded by the channel coherence bandwidth. In fact, from Eq. (7) and thanks to Parseval's identity, $R_{y}^{q \alpha_{0}}(N)$ can be expressed as

$$
R_{y}^{q \alpha_{0}}(N)=R_{x}^{q \alpha_{0}}(N) \int_{-\frac{1}{2}}^{\frac{1}{2}} \mathbb{E}\left[H(\nu) H^{*}\left(\nu-q \alpha_{0}\right)\right] d \nu
$$

where $H(\nu)=\sum_{l=0}^{L} h(l) e^{-2 i \pi l \nu}$. Thus, from the definition of S (see Eq. (2)), the cycle frequencies used to estimate the signal power have to be limited to the case where $R_{y}^{q \alpha_{0}}(N) \simeq$ $R_{x}^{q \alpha_{0}}(N) \sum_{l=0}^{L} \sigma_{h(l)}^{2}$ which is equivalent to choose $q \alpha_{0}$ within the coherence bandwidth where $\mathbb{E}\left[H(\nu) H^{*}\left(\nu-q \alpha_{0}\right)\right] \simeq$ $\mathbb{E}\left[|H(\nu)|^{2}\right]$. The coherence bandwidth $B_{c}$ is usually defined thanks to the channel root mean square delay spread [8]. In our case, as the channel impulse response is unknown at reception, $B_{c}$ is approximated as $\hat{B}_{c}=1 /(\beta \hat{L})$ where $\beta$ is a coefficient expressing the desired correlation rate within $B_{c}$. Consequently, we choose $N_{c}=\min \left(\frac{N+D}{\beta \tilde{L}}, \frac{N}{2 D}\right)$. As shown in section $\mathrm{V}, \beta$ 's choice has only a very little influence on the estimator performance.

\section{Simulations}

In the following, all the results are averaged over 10000 Monte Carlo runs. Fixed WiMAX signals [9] are simulated. We recall that $N=256$ and choose $D=32$. The propagation channel is simulated using the SUI-4 model ${ }^{2}$ defined as

\footnotetext{
${ }^{2}$ The Doppler of the SUI-4 model as defined in [10] is not simulated here as it is very small $(\simeq 0.2 \mathrm{~Hz})$ in comparison with usual WiMAX frequency ranges. Thus, the channel can be considered as time-invariant over the observation windows tested in this section.
} 
TABLE I

SUI-4 CHANNEL MODEL

\begin{tabular}{|c|c|c|}
\hline Path number & Delay $(\mu \mathrm{s})$ & Power $(\mathrm{dB})$ \\
\hline 1 & 0 & 0 \\
2 & 1.5 & -4 \\
3 & 4 & -8 \\
\hline
\end{tabular}

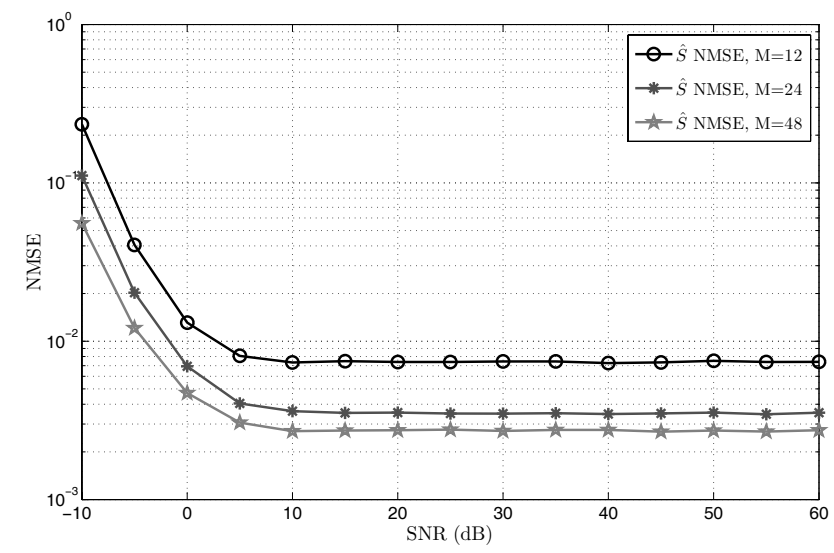

Fig. 2. NMSE of the signal power estimator.

Figure 1 shows the Normalized Mean Square Error (NMSE) of the noise variance estimation versus SNR for various observation window lengths. The NMSE is here defined as $\mathbb{E}\left[\left({\hat{\sigma_{\eta}^{2}}}^{2}-\sigma_{\eta}^{2}\right)^{2}\right] / \sigma_{\eta}^{4}$. We observe that the performance is significantly improved as the number of available OFDM symbols increases. Moreover, for SNR below $20 \mathrm{~dB}$, the estimator defined in Eq. (5) occasionally under-estimates $L$ which results in performance degradation for $-5 \leq \mathrm{SNR}<20$. However, this under-estimation is beneficial for very low SNR. In this case, as the noise power is strongly predominant, $J(u)$ (see Eq. (3)) is a also a valid noise variance estimator for $u<L$. The number of samples used for this estimator is therefore greater than in the case where $L$ is perfectly known (i.e. $>(D-L) M$ ) which results in better performance.

In Fig. 2, we plot the NMSE of the signal power estimation versus SNR for different $M . \mathrm{NMSE}=\mathbb{E}\left[(\hat{S}-S)^{2}\right] / S^{2}$ and $\beta$ is set to 5 . The observation window impacts the performance in a similar way to the noise variance estimator. Note that the relative performance improvement diminishes quickly as $M$ increases.

Figure 3 compares the SNR performance of the approximate maximum likelihood (AML) estimator described in [3] with our proposed method. Figure 3 highlights two limitations of the AML algorithm. First, as previously explained, this method depends on a subjective threshold, denoted $\alpha$ in [3], that has a strong impact on the performance. Then, as the signal power and noise variance estimations are not independent, the SNR estimation gets deteriorated at low and high SNR. Moreover, Fig. 3 reveals that the algorithm presented in this paper globally outperforms the AML criterion and that the coherence bandwidth choice (i.e. $\beta$ ) has little influence on the estimator performance.

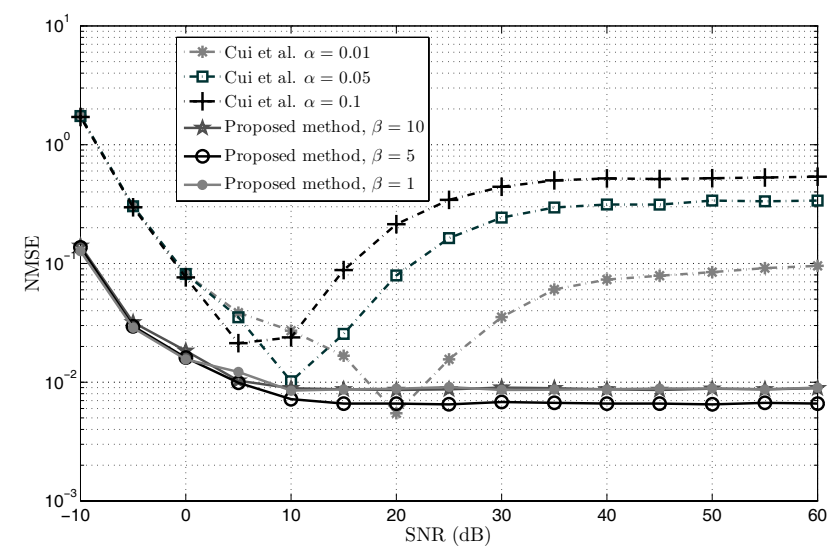

Fig. 3. Performance comparison between the proposed method and the AML method [3], $\mathrm{M}=24$.

\section{CONCLUSION}

In this contribution, we presented a new method based on the use of the cyclic prefix to estimate the SNR of OFDM signals without the knowledge of any pilots. We exploited the correlation induced by this prefix as well as its periodicity to respectively derive noise variance and signal power estimators. Simulations indicate that the proposed algorithm exhibits good results and outperforms the approximate maximum likelihood method described in [3].

\section{REFERENCES}

[1] H. Xu, G. Wei, and J. Zhu, "A novel SNR estimation algorithm for OFDM," in Proc. IEEE Vehicular Technology Conf., vol. 5, June 2005, pp. 3068-3071.

[2] W. Yi, L. Lihua, Z. Ping, L. Zemin, and Z. Yu, "A new noise variance estimation algorithm for multiuser OFDM systems," in Proc. IEEE Conf. on Personal, Indoor and Mobile Radio Communications, Sept. 2007, pp. $1-4$.

[3] T. Cui and C. Tellambura, "Power delay profile and noise variance estimation for OFDM," IEEE Commun. Lett., vol. 10, no. 1, Jan. 2006.

[4] J. van de Beek, M. Sandell, and P. Borjesson, "ML estimation of time and frequency offset in OFDM systems," IEEE Trans. Signal Processing, vol. 49, pp. 1800-1805, July 1997.

[5] B. Park, E. Ko, H. Cheon, C. Kang, and D. Hong, "A blind OFDM synchronization algorithm based on cyclic correlation," in Proc. IEEE Global Telecommunications Conf., vol. 5, Nov. 2001, pp. 3116-3119.

[6] P. Jallon, "An algorithm for detection of DVB-T signals based on their second order statistics," EURASIP J. Wireless Commun. and Networking, vol. 2008, 2008, article ID 538236.

[7] W. A. Gardner, A. Napolitano, and L. Paurac, "Cyclostationarity: half a century of research," Signal Processing, vol. 86, no. 4, pp. 639-697, Apr. 2006.

[8] T. Rappaport, Wireless Communications: Principles and Practices. Prentice Hall, 2002.

[9] Part 16: Air Interface for Fixed Broadband Wireless Access Systems, IEEE Std. 802.16, 2004.

[10] V. E. et al., Channel Models for Fixed Wireless Applications, IEEE 802.16 Broadband Wireless Access Working Group, 2001. 